\title{
Research Square \\ Influence of KI Salt Concentration on The Hydroxypropyl Methylcellulose Films: Optical Study
}

Sannappa J ( $\square$ sannappaj2012@gmail.com )

Kuvempu University https://orcid.org/0000-0002-4677-5341

\section{Thippaiah Demappa}

University of Mysore

Sunilkumar

Kuvempu University

\section{Research Article}

Keywords: Hydroxypropyl methylcellulose (HPMC) salt-composite, Absorption band, bandgap, optical dielectric function, optical conductivity, refractive index

Posted Date: August 4th, 2021

DOI: https://doi.org/10.21203/rs.3.rs-770530/v1

License: (c) (1) This work is licensed under a Creative Commons Attribution 4.0 International License. Read Full License 


\title{
Influence of KI salt concentration on the Hydroxypropyl methylcellulose films: Optical study
}

\author{
Sunilkumar $^{1,3} \cdot$ Thippaiah Demappa $^{2} \cdot$ J Sannappa $^{3, *}$ \\ ${ }^{1}$ Department of Physics, S.S Arts College and T.P Science Institute, Belagavi 591313, India \\ ${ }^{2}$ Department of Polymer Science, Sir MV, P G Centre, University of Mysore, Mandya 571402, India \\ ${ }^{3}$ Department of P G Studies and Research in Physics, Jnana Sahyadri, Kuvempu University, Shimoga 577401, \\ India \\ * Corresponding author : sannappaj2012@gmail.com
}

\begin{abstract}
Herein the optical properties of Hydroxypropylmethyl cellulose/Potassium iodide (HPMC/KI) composite films were determined. Casting technique was introduced to make HPMC/KI films with different KI salt concentrations (0.1-1) wt $\%$. The absorbance model was used to determine parameters like absorption edge, refractive index, real and imaginary sections of dielectric function, extinction coefficient, Urbach energy, band gap and optical conductivity in the spectral range $200-800 \mathrm{~nm}$. As shown by study, KI salt doping affects the optical properties of HPMC. The absorption edge $\left(E_{e}\right)$ was widely displaced towards a region of lower photonic energy. For the 1 wt $\% \mathrm{KI} / \mathrm{HPMC}$ film, the direct and indirect optical bandwidth gaps of pure HPMC were reduced from $5.6 \mathrm{eV}$ to $2.56 \mathrm{eV}$ and 5.86 to $2.5 \mathrm{eV}$ respectively. The optical dielectric loss method was effectively employed as an alternate method for estimating the optical bandgap. In addition, the Tauc's extrapolation method identified the kind of electronic transition. The variation of optical energy band gap and dielectric constant based on KI salt concentration was used to investigate the credibility of the Penn's model. In salt-composite films, an increase in Urbach energy and optical conductivity were observed which may be evidence of large shift from tail-to-tail and band to tail. Meanwhile, X-ray diffraction (XRD) examination revealed that the KI salts damaged the HPMC polymer's crystalline phase. Lastly, the films were also subjected to Fourier transform infrared spectroscopy (FTIR). The considerable variation in transmittance and band change in FTIR spectra was exposed for doped films.
\end{abstract}

Keywords Hydroxypropyl methylcellulose (HPMC) salt-composite · Absorption band · bandgap · optical dielectric function $\cdot$ optical conductivity $\cdot$ refractive index 


\section{Introduction}

Doping is the process of adding impurities into a host polymer to modulate its physical properties like electrical, optical, thermal, mechanical, and structural properties. The comprehensive investigation of the doped polymer with various concentrations of dopant enables the selection of the desired properties $[1,2]$. Depending on their reactivity with the host matrix, dopants can be used to modify the electrical and optical properties of polymers [3].

The effect of doping concentration on optical, morphological and dielectric properties of PMMA films has been studied by L. N. Ismail et al [4]. Ranganath M R et al [5,6] studied optical properties of iodine and ferric chloride $\mathrm{FeCl}_{3}$ doped poly(vinyl alcohol) - poly(vinyl pyrrolidone) ( PVA/PVP) blend films. The doping of HPMC with $\mathrm{FeCl}_{3}$ in aqueous dispersions has been studied by $\mathrm{Y}$ Prakash et al [7], which showed that doping of $\mathrm{FeCl}_{3}$ caused a significant change of Physico-mechanical, AC-conductivity, and microstructural properties. N Sandhya Rani et al [8-10] studied the effect of $\mathrm{CdCl}_{2}$ and $\mathrm{NaI}$ on the functional, structural, electrical conductivity and thermal properties of HPMC polymer electrolyte films. Biodegradable polymers are raising prominence as a green alternative to commercially available non-degradable polymers. They are becoming a growing discipline of research and have attracted many industries as they are plentiful and renewable $[11,12]$. The majority of conjugated polymers with intramolecular charge transfer (ICT) properties have been reported to be narrow bandgap polymers

[13]. But conjugated polymers have some limitations such as poor efficiency and rapid degradation [14]. On the other hand, polar polymers like HPMC, methylcellulose (MC), and chitosan are low cost, good film-forming ability and have long lifetimes. The HPMC polymer has opted for its pleasant characteristics. HPMC is a cellulosebased biopolymer, eco-friendly, soluble in both water and polar organic solvents and has an excellent film-forming property with a different class of metal ions, as well as its ability to store charge [8-10,15-19]. HPMC shows a semi-crystalline complexion that resulted in two structures (crystalline and amorphous). The crystalline structures deliver solid mechanical support/backing for polymer electrolytes. While, the amorphous phase boosts the electric and optical properties in the host polymer [9]. The optical and electrical nature of polymers have piqued interest due to their contributions in optical and optoelectronic devices with remarkable polarisation, antireflection, reflection, and interference properties. Polymer-based optical communications like optical waveguides, optical connectors, and optical fibers are fruitful than silica-based optical materials, on account of their easy processing and mass production at relatively low cost [19-21]. Finding a proper bandgap material is one of the most difficult tasks for photovoltaic and optoelectronic applications [22]. Prior studies have reported that functional materials, composites, and organic polymers with sufficient optical bandgaps are valuable for the organic light-emitting diodes (OLED), photonics, and optoelectronic products [23-25]. 
A comparatively small number of studies have been reported on the development of polymeric electrolyte systems based on HPMC. There are a few articles on $\mathrm{HPMC}$ uses $\mathrm{NaI}, \mathrm{CdCl}_{2}, \mathrm{FeCl}_{3}, \mathrm{NaF}$ and $\mathrm{NaCl}[8,9,26]$. Most of the researchers focused on the studies on lithium salt in polymer systems because it has high energy density and rechargeability benefits. But due to high moisture sensitivity, it is difficult to maintain atmospheric conditions. $\mathrm{KI}$ is the most economic, significant iodide compound and a salt of stable iodine. The $\mathrm{KI}$ is a prominent material regarding its wide-ranging applications. Potassium is perhaps more economic and plenteous than lithium. Potassium is now extra resistive to moisture than lithium [27,28]. While, only a few studies on potassium complexes polymer electrolytes have been done. N Krishna Jyothi et al studied electrolytes for electrochemical and solid-state battery applications of PAN-KI complexed gel polymer electrolytes [27,28]. But no merit has been given to potassium salt complexed HPMC-based polymer electrolytes. Accordingly, the study has been based on potassium salts.

The really important tool for determining band structure, optical constants and electronic properties of pure and doped polymers is the optical spectrum [20]. UV/VIS spectrometer was successfully used to examine the impact of KI salt on the optical properties of HPMC films, which provides evidence for estimating various optical parameters. KI offers excellent amorphous and compatible temper to the HPMC. HPMC has a potential advantage in optical storage systems. Which can still be shaped by adding KI salt.

In the view of the usefulness of HPMC and KI salt, this research was carried out to prepare water-soluble HPMC/KI film composites featuring great film-forming capacity, small optical bandgap, transparency, and good optical conductivity by employing the solution cast technique. The prepared films have been characterized by various analytical techniques like XRD, FT-IR and UV-VIS.

\section{Materials and methods}

Potassium Iodide (KI) and Hydroxypropyl methylcellulose (HPMC-E15LV) were used in our experiment. KI used in this work was obtained in the salt form and HPMC was obtained in the powder form. All chemical constituents were used of analytical grade purchased from Sigma Aldrich. Polymer composite films were primed by a solution casting method treating double distilled water as a solvent [29]. In this experimental process, initially three grams (3gm) of HPMC were added into $100 \mathrm{~mL}$ de-ionized water ( $3 \mathrm{wt} \%)$ for 30 minutes slowly stirring the solution with a magnetic stirrer at room temperature $\left(\sim 30^{\circ} \mathrm{C}\right)$ for total dissolving. Also, pure HPMC with a suitable weight percentage ratio ( $0.1 \mathrm{wt} \%, 0.3 \mathrm{wt} \%, 0.5 \mathrm{wt} \%$ and $1 \mathrm{wt} \%)$ of $\mathrm{KI}$ salt were added (dissolved) into each prepared $3 \mathrm{wt} \%$ (100mL) of HPMC polymeric solution under continual stirring for 10 to $12 \mathrm{hrs}$. After all, viscous 
solutions were transferred into flattened glass plates/Petri dishes $(10 \mathrm{~cm} \times 15 \mathrm{~cm})$ and kept in a vacuum chamber for evaporation for 5 days. Finally, the universal solvent water was let to vaporise freely at room temperature conditions to achieve polymer films at the base of glass plates. The film samples were collected in high-discharge desiccators to prevent dust and moisture uptake. The pure HPMC film and different weight percent ration of KI salt was obtained, (B) HPMC film, (B1) HPMC: $0.1 \mathrm{wt} \%$ KI, (B2) HPMC: $0.3 \mathrm{wt} \% \mathrm{KI}$, (B3) HPMC: $0.5 \mathrm{wt} \% \mathrm{KI}$ and (B4) HPMC:1 wt\% KI. The thickness of these films was fixed using a Screw gauge by measuring the thickness at five different parts of each film sample and was found to be approximately $300 \mu \mathrm{m}$.

\subsection{Characterization techniques}

After preparation of HPMC and HPMC:KI complex polymer film, samples were evaluated by different experimental techniques, i.e. XRD, FT-IR and UV-Vis spectroscopy. The crystallinity study of prepared polymer blend film was assessed by Rigaku Miniflex-II X-ray diffractometer through Ni filtered, CuK $\alpha$ radiation $(\lambda=1.540$ $\AA$ A). The diffraction patterns were documented at Bragg's angle $(2 \Theta)$ in the scale of $10^{\circ}-80^{\circ}$ with a scanning speed of $5^{\circ}$ min and step size $0.02^{\circ}$. The chemical composition was explored by employing a Fourier transform infrared (FT-IR) with model-ALPHA BRUKER spectrometer and analysis was performed in the frequency scale between $4000-500 \mathrm{~cm}^{-1}$ with $4 \mathrm{~cm}^{-1}$ resolutions. The optical properties of all the samples were assessed by a double-beam Ultraviolet-Visible (UV-Vis) spectrometer (Lambda 35)

\section{Results and discussion}

\subsection{Transmittance and Absorption Analysis}

UV-VIS spectroscopy was used to examine the optical characteristics of HPMC polymer films. The plot of transmittance for virgin HPMC and loaded HPMC with different KI salt wt\% is shown in Fig. 1 which shows that the virgin HPMC achieved remarkable transparency of around $90 \%$ outside the visible province whereas the transparency declined in the lower zone. However, increasing salt concentration resulted in a change in transparency. That is because of the change in refractive index due to scattering inside the doped films [30]. The dopant is accountable for variation of transparency. However, one of the biggest challenges in designing composite materials for optical applications is maintaining transparency [31]. Significantly, the transparency of the doped samples in the visible range was found to be greater than $85 \%$. The pure HPMC showed shoulder-like behaviour and it was shifted to a higher wavelength in the doped samples. This is due to the interaction of dopant with the host polymer matrix. We will go over this in further detail in the following post. 


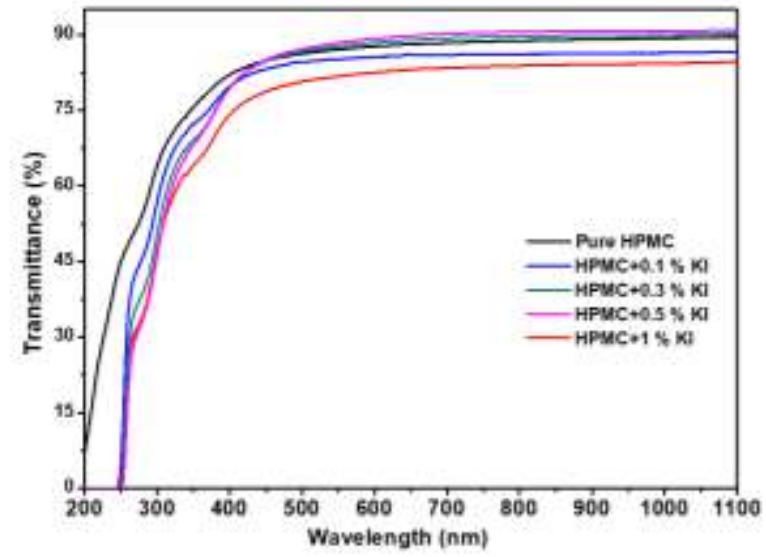

Fig. 1 Pure and doped HPMC samples transmittance spectrum.

Figure 2 displays absorbance spectra for doped and undoped films in the $200-700 \mathrm{~nm}$ range. This shows that no absorption band was seen in the visible range and the HPMC film is transparent in the visible light. The virgin HPMC contains a slight hump at $275 \mathrm{~nm}$ that can be seen due to the $\mathrm{n} \rightarrow \pi^{*}$ transition in the methyl groups [32]. The shift in the humps for the samples once doping with KI specify a significant interaction between HPMC and KI [33]. The absorption spectra declined with rising wavelength for Pure and doped samples. According to Beer's Law, absorption increased as the KI doping concentration increased [33,34,35]. The quantity of absorbing molecules determines the degree of absorption. This is consistent with prior reports and findings [36].

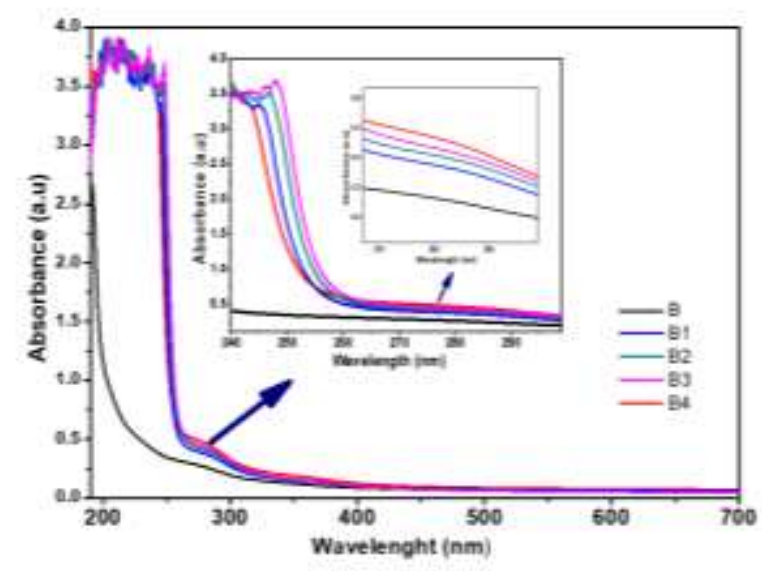

Fig. 2 Pure and doped HPMC samples absorbance spectrum. 
The pure HPMC and HPMC/KI films optical absorption coefficient $(\alpha)$ versus wavelength $(\lambda)$ can be assessed via the subsequent relation (1) [37]:

$$
\alpha=\frac{1}{d} \ln \left(\frac{1}{T}\right)=\frac{2.303 A}{d}
$$

Where, $\mathrm{A}$ is the absorbance, $\mathrm{T}$ is the transmittance and the thickness of the film is $\mathrm{d}$. The photon energy hv dependent optical absorption coefficient was revealed in Fig. 3 for pure and doped HPMC samples. The basic absorption edge is thought to be the really important properties of the absorption spectra of crystalline and noncrystalline substances [38]. Extrapolating the linear section of the curves to zero absorption $(\alpha=0)$ yielded the position of the absorption edge. The absorption coefficient increased largely near the edge due excitation of electrons from the lower energy band to higher energy [39]. The virgin HPMC's absorption edge $\left(E_{e}\right)$ was discovered to be $5.09 \mathrm{eV}$ and reduced to $2.57 \mathrm{eV}$ for $1 \mathrm{wt} \%$ of KI fixed HPMC films (reported in Table 1). The establishment of intramolecular charge transfer (ICT) and enrichment in intermolecular stacking is responsible for such a large change in the absorption edge in the doped HPMC polymer films [40]. The relocation of the absorption edge $\left(E_{e}\right)$ to smaller photon energy indicates that the optical bandgap has shortened in the doped samples [41]. The lowest absorption edge of $2.57 \mathrm{eV}$ was found in the highest doped polymer indicating that it is the most suitable conduction among the other films.

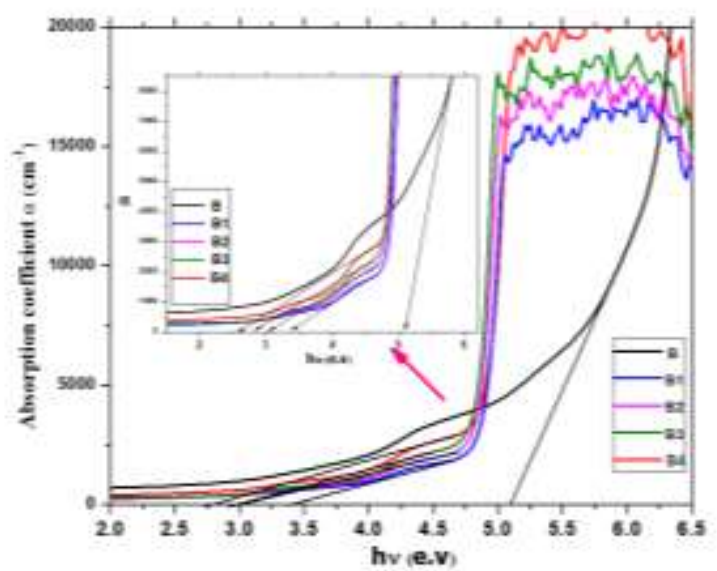

Fig. 3 Pure and doped HPMC samples absorption coefficient ( $\alpha$ ) vs photon energy (hv).

\subsection{Tail study}

The feature of the primed polymer is predicted through Urbach energy. In the shorter absorption region, the absorption coefficient $(\alpha)$ has an exponential effect on photon energy (hv) and complies the Urbach relation (2) [42]: 


$$
\alpha=\alpha_{o} \exp \left(\frac{h \omega}{E_{g}}\right)
$$

The Urbach energy is also known as band tail energy, is denoted by $E_{b}$ and $\alpha_{o}$ denotes a constant [43]. Figure 4 depicts the graph of $\ln (\alpha)$ vs photon energy hv for all samples. The values of Urbach energy $\left(E_{b}\right)$ for different doping concentrations can be computed from the slopes of the linear portion of the plot and are listed in Table 1. The optical bandgap is inversely proportional to the Urbach energy. It has been proven that the increase of Urbach energy leads to an increase of structural disorder in the polymer complexes. As a result, the states will be redistributed from band to tail, allowing a larger number of states to participate in band-to-tail and tail-to-tail transfers [44]. The measured Urbach energy $\left(E_{b}\right)$ for the pure HPMC sample was $556 \mathrm{meV}$ and improved to 874 meV for the doped HPMC film with $1 \mathrm{wt} \%$ of the KI. Early studies have shown that a decrease in the crystalline province may increase Urbach energy. This confirms that the trap states are increasing inside the optical energy bandgap and a reduction in the optical bandgap is possible $[43,45,46]$.

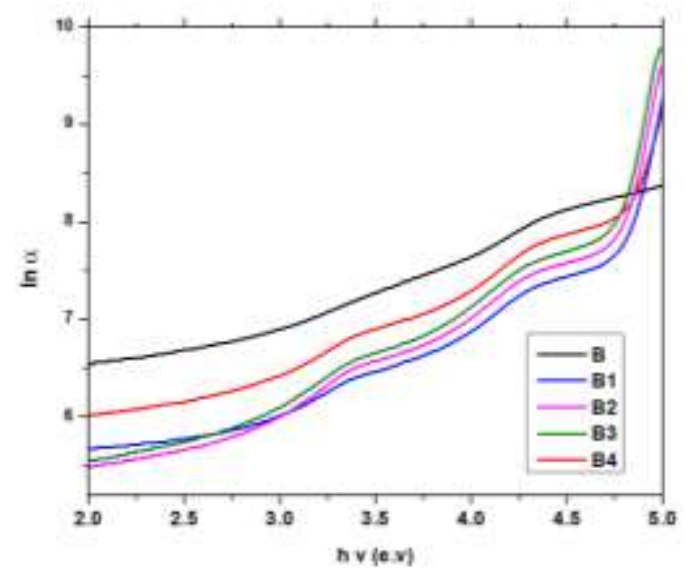

Fig. 4 Urbach plots for pure HPMC and doped HPMC samples.

\subsection{Bandgap Study}

\subsubsection{Tauc and Optical dielectric models}

The optical absorption of the films to be learned at a great deal to gain more knowledge about the band structure [47]. The fundamental absorption due to transition of electrons from the valence band to the conduction band can be utilised to fix the bandgap of a substance [48]. The optical energy band gap of HPMC and HPMC/KI composites were assessed using Davis and Mott solution, which gives the connection between the incident photon energy (hv) and absorption coefficient ( $\alpha$ ) by the Tauc plot equation (3) [49]. 


$$
\alpha=A\left(h v-E_{g}\right)^{r}
$$

Where, $\mathrm{A}$ is the slope of the Tauc edge known as band tail parameter, $E_{g}$ denotes the optical energy bandgap and $r$ denotes the exponent factor. The exponent $r$ gives the nature of electronic transition accountable for absorption and it follows the values of $1 / 2,2,3 / 2$ and 3 for permitted direct, allowed indirect, forbidden direct and forbidden indirect electron excitations respectively [48].

Table 1 Optical parameters of pure and doped HPMC samples

\begin{tabular}{ccccccc}
\hline HPMC/KI & Sample & Absorption & Urbach & Direct & Indirect & Estimated \\
wt\% & Code & edge & energy & band gap & band gap & bandgap from \\
& & $\boldsymbol{E}_{\boldsymbol{e}}(\mathbf{e . v})$ & $\boldsymbol{E}_{\boldsymbol{b}}(\mathbf{m e v})$ & $\boldsymbol{E}_{\boldsymbol{d}}(\mathbf{e . v})$ & $\boldsymbol{E}_{\boldsymbol{i m \boldsymbol { d }}}(\mathbf{e . v})$ & $\boldsymbol{\varepsilon}_{\mathbf{2}}$-hv graph \\
\hline Virgin & $\mathrm{B}$ & 5.09 & 556 & 5.60 & 5.86 & 5.54 \\
0.1 & $\mathrm{~B} 1$ & 3.39 & 758 & 2.95 & 3.16 & 2.85 \\
0.3 & $\mathrm{~B} 2$ & 3.00 & 760 & 2.87 & 2.98 & 2.73 \\
1 & $\mathrm{~B} 3$ & 2.83 & 862 & 2.79 & 2.69 & 2.64 \\
\hline
\end{tabular}

The variation of $(\alpha h v)^{2}$ and $(\alpha h v)^{1 / 2}$ as a function of photon energy (hv) at room temperature for pristine and KI doped HPMC films are shown in Figures 5 and 6 respectively. The linear region of curves may be related to the direct and indirect transitions between the valance and conduction bands [50]. The $E_{d}$ and $E_{\text {ind }}$ can be estimated by extrapolating this linear region to zero absorption. The $E_{d}$ and $E_{\text {ind }}$ readings are given in table 1 . The direct energy bandgap $\left(E_{d}\right)$ and indirect energy bandgap $\left(E_{\text {ind }}\right)$ of composite films are decreased from $5.60 \mathrm{eV}$ to $2.56 \mathrm{eV}$ and $5.86 \mathrm{eV}$ to $2.50 \mathrm{eV}$ respectively. This indicates that the rise of the KI percentage makes HPMC films more semiconducting [51]. The assembly of KI particles in the electronic structure of the matrix of HPMC may be responsible for the development of localised states between the valence and conduction bands, resulting in the decline of optical energy band gap [52]. It was noticed from $E_{d}$ and $E_{\text {ind }}$ values that the rise in the percentage of KI encourages a change in the morphology and the microstructure of the HPMC matrix. This change may be due to structural disorder in the HPMC amorphous region [53]. Prior studies have reported that functional materials, composites, and organic polymers with sufficient optical bandgaps are valuable for the organic light-emitting 
diodes (OLED), photonics, and optoelectronic products [23-25]. The HPMC/KI films fine optical bandgap shows their suitability for optoelectronics, solar cells and photonics applications.
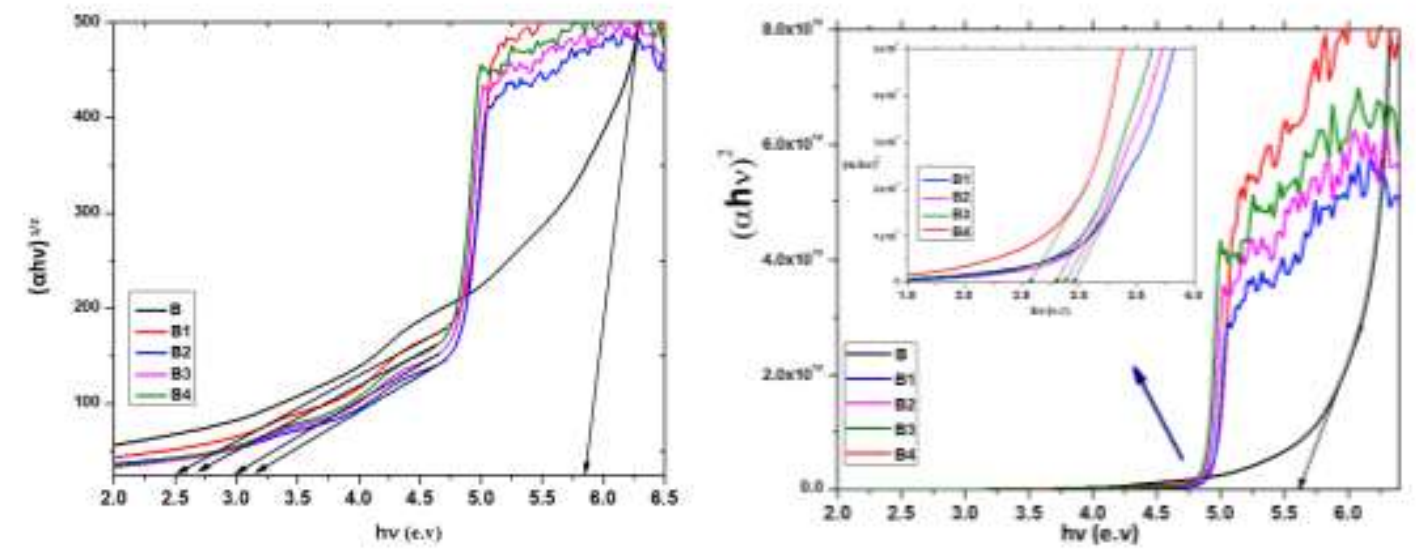

Fig. $5(\alpha h v)^{2}$ vs photon energy (hv) for pure and doped HPMC films.

Fig. $6(\alpha h v)^{1 / 2}$ vs photon energy (hv) for pure and doped HPMC films.

\subsection{Refractive Index Study (n):}

Another important optical constant is the refractive index (n), which offers knowledge about local fields, polarisation, and phase velocity of light in propagating substance and is used to design optical devices. The following equation (4) can be used to calculate it [54].

$$
\mathrm{n}=\frac{1}{T_{S}}+\sqrt{\frac{1}{T_{S}-1}}
$$

Where $\mathrm{T}_{\mathrm{S}}$ is the percentage transmission coefficient. Figure 7 illustrates that as the wavelength increases and refractive index drops, suggesting proper dispersion behavior [55]. While this aspect of dispersion is critical for practical drives and optical system design [30]. The Refractive index for complex polymer shows a small hump around $370 \mathrm{~nm}$ and nearly flat behavior between $400-700 \mathrm{~nm}$. The observable humps may be attributable to the formation of $\mathrm{KI}$ in the host polymer matrix. In the visible region, $\mathrm{n}$ does not equal zero indicating that some incident photon light may be absorbed. 


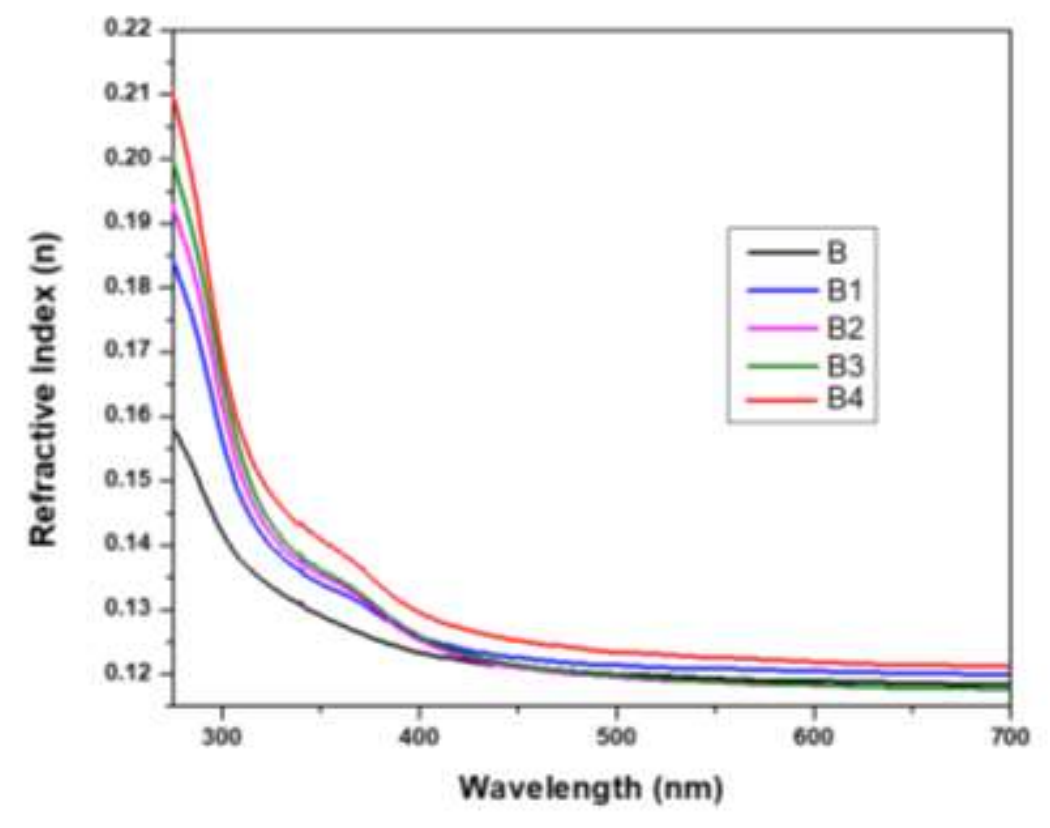

Fig. 7 refractive index (n) vs wavelength for pure and doped HPMC samples.

\subsection{Extinction coefficient (K)}

The extinction coefficient $(\mathrm{K})$ determines how much light is reduced due to scattering and absorption per unit distance of penetration medium.

The extinction coefficient $\mathrm{K}$ of virgin and doped samples varies with the wavelength in the 200-600 nm region as seen in Fig. 8(a). Extinction coefficient (K) values are higher at shorter wavelengths $(200-400 \mathrm{~nm})$ indicating considerable absorption in this region. It is undeniable that the extinction coefficient for pure HPMC and doped samples decreases with an increase in wavelengths. Because the following equation (5) [29,30] shows that the extinction coefficient is directly proportional to the absorption coefficient. Fig. 1 for $\alpha$ and Fig. 4 for $\mathrm{K}$ follow the same pattern.

$$
K=\frac{\lambda \alpha}{4 \pi}
$$

Where, $\alpha$ is the absorption coefficient. Figure 8(a) and 8(b) represents the dependence of extinction coefficient on wavelength and photon energy respectively. The increase in extinction coefficient with a raise in 
photon energy (decrease wavelength) stating that a fraction of light reduced due to scattering, and absorbance raises [56].
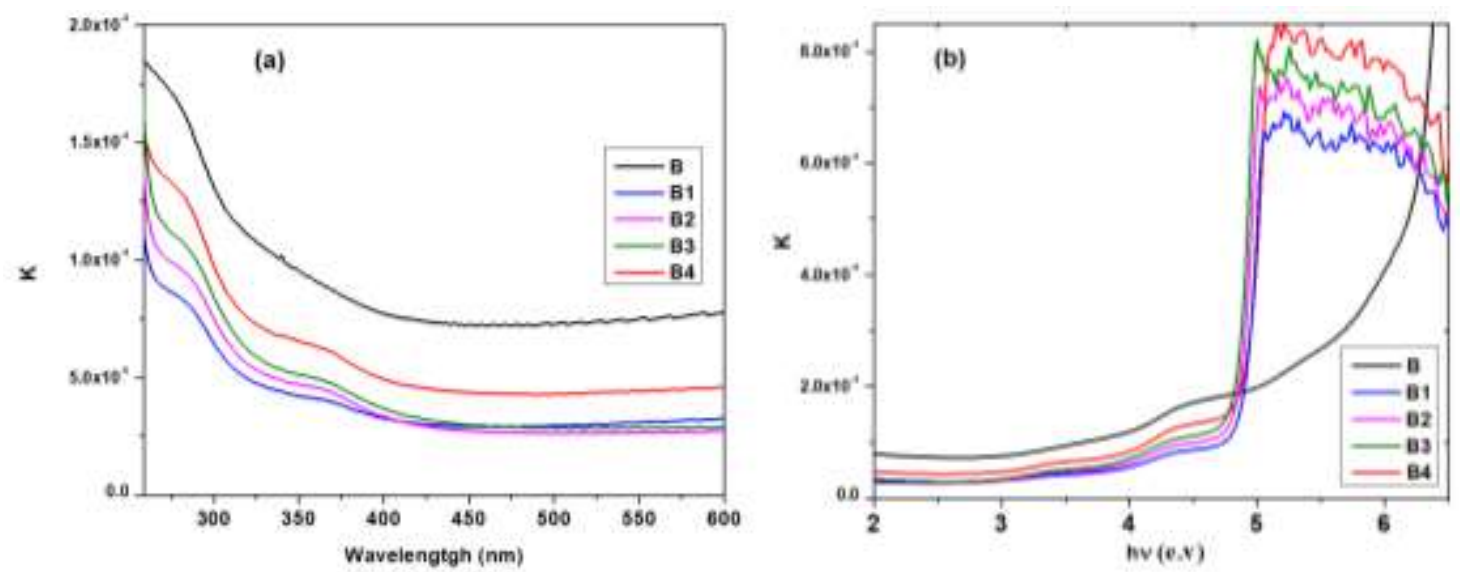

Fig. 8 Extinction coefficient variation as a function of (a) wavelength and (b) energy.

\subsection{The real $\left(\varepsilon_{1}\right)$ and imaginary $\left(\varepsilon_{2}\right)$ parts of the dielectric function $\left(\varepsilon^{*}\right)$}

The linear dependence of the substance to electromagnetic radiation can be understood with a complex dielectric function $\left(\varepsilon^{*}=\varepsilon_{1}-\mathrm{i} \varepsilon_{2}\right)$, which will give you a better knowledge of the solid's optical features. Equations (6) and (7) can be used to compute the real and imaginary parts of dielectric constants.

$$
\begin{aligned}
& \varepsilon_{1}=n^{2}-K^{2} \\
& \varepsilon_{2}=2 n k
\end{aligned}
$$

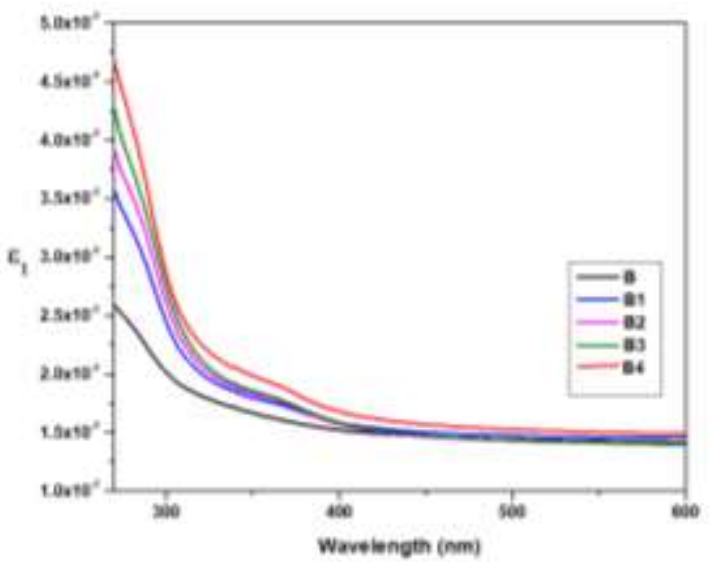

Fig. 9 Photon energy vs optical dielectric constant for pure and doped HPMC samples.

The most significant physical variable that hangs on the bandgap is the dielectric function's real part, that relates to an electronic part [57]. The effect of the real component of the dielectric constant on wavelength is seen 
in Fig. 9 for both pure and doped films. With rising wavelength and impurity concentration, the real part of the curve grows and shifts the curve's vertex towards higher wavelengths may be credited to equation (3) by the real part of the dielectric constant with refractive index [58]. The largest value of optical dielectric constant $\varepsilon_{1}$ for the smallest optical band gap justifies the well-known Penn's model [59].

The imaginary part $\varepsilon_{2}$ is directly linked to the valence and conduction band, and it refers to the material's optical absorption which is given by (4). The optical dielectric loss versus directed photon energy and wavelength for all films is shown in Figures 10(a) and 10(b) respectively. In low photon energy region, the dielectric loss was found to be relatively small but exponentially raised at high photon energy (i.e at lower wavelength) for the pure and doped HPMC film. On the other hand, the absorption edge in doped films was noticed to be reallocated to the lower photon energy. It has been proved that the absorption edge resulting from dielectric loss should be highly comparable to the Tauc's relation anticipated values [60]. Table 1 shows the calculated values of the bandgap using both approaches (optical dielectric loss and Tauc).

The optical bandgap results obtained in Fig. 10(a) are similar to those obtained in Fig. 6. Therefore, the optical dielectric function can be utilized to calculate the optical bandgap and analyse the band structure [30,41]. Based on the preceding analysis, it is evident that the Tauc's model can be used to fix the mode of electronic transition and optical dielectric loss can be utilised to analyse the bandgap and $[41,43]$.
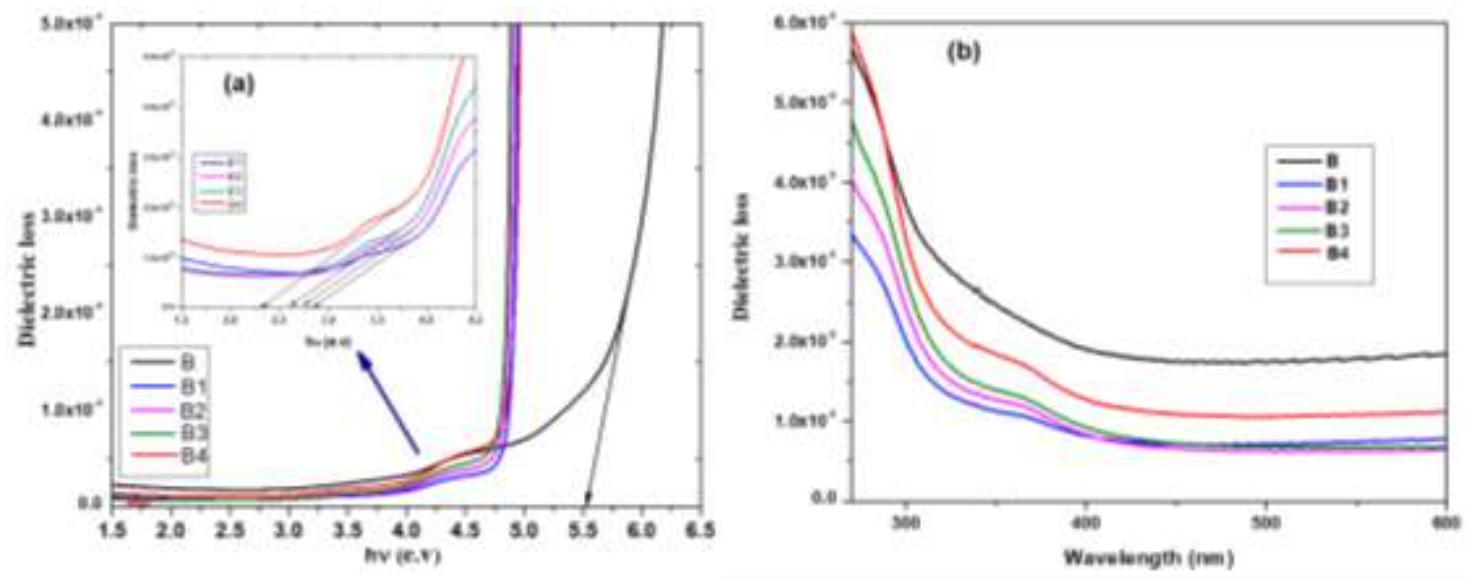

Fig. 10 Optical dielectric loss vs photon energy (hv) for pure and doped HPMC samples.

\subsection{Optical Conductivity:}


The optical conductivity $\sigma_{o p t}$ of a material is one of the most essential characteristics that affect its optical properties. It is used to expose the material's interband allowed optical transitions. The optical conductivity $\sigma_{o p t}$ determined by relation (8) using the absorption coefficient $(\alpha)$ and refractive index (n) of the material $[49,61]$.

$$
\sigma_{o p t}=\frac{\alpha n c}{4 \pi}
$$

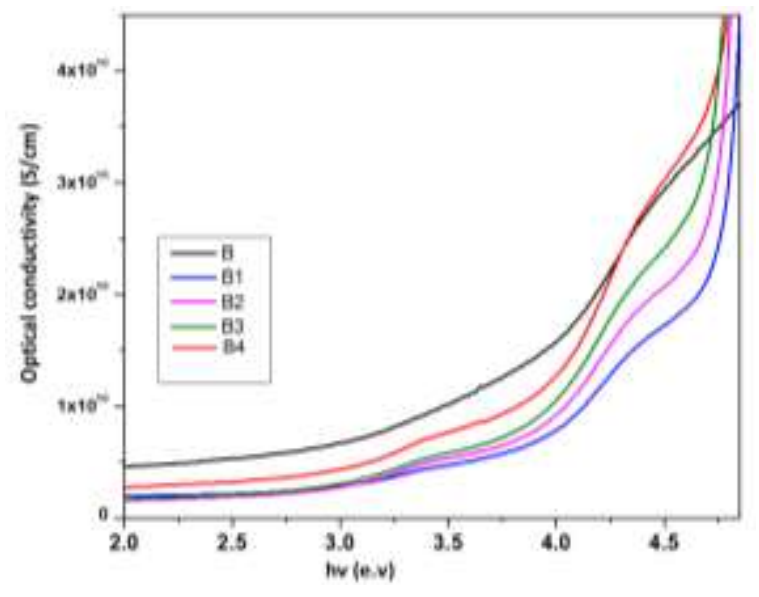

Fig. 11 The optical conductivity as a function of photon energy (hu) for pure and doped HPMC.

For pure and doped films, Fig. 11 displays the optical conductivity versus photon energy. The optical conductivity increases as photon energy increases. The increase in absorption coefficient is responsible for the excitation of carriers from the valence to conduction band due to the absorption of photon energy given by equation 8 . The absorption coefficient and refractive index have a direct relationship with optical conductivity and shadows the same fashion of $\sigma_{o p t}$.

\subsection{XRD Study}

The XRD patterns of pure HPMC and doped HPMC samples are shown in Fig. 12. It is known that a large crystalline hump appears at around $2 \theta=20.88^{\circ}$ for pure HPMC polymer [2]. KI also showed its peaks (PCPDF file no. 780750). The intensity of broad peak suggestively declines for all doped film samples $(0.1 \mathrm{wt} \%$ to $1 \mathrm{wt} \%$ KI) as shown in Fig. 12. The calculated crystallinity percentage was also decreased with dopant concentration (given in table 2) which signifies the distribution of KI in the host polymer's matrix [27,28]. According to the XRD results, the amorphous segment increases in the salt complex samples implying that disorder is more prevalent in doped samples. Previous studies have shown that an increase in the crystalline region in a sample can indicate a drop in the Urbach energy, whereas an increase in the amorphous 
area is indirectly linked to an increase in the Urbach energy [30,43,45,46]. As a consequence, both amorphous percentage and Urbach energy improved with dopant concentration. XRD measurements found in this mixture will support the Urbach energy calculation $(\mathrm{Eu})$.

Table 2 XRD for pure and doped HPMC samples

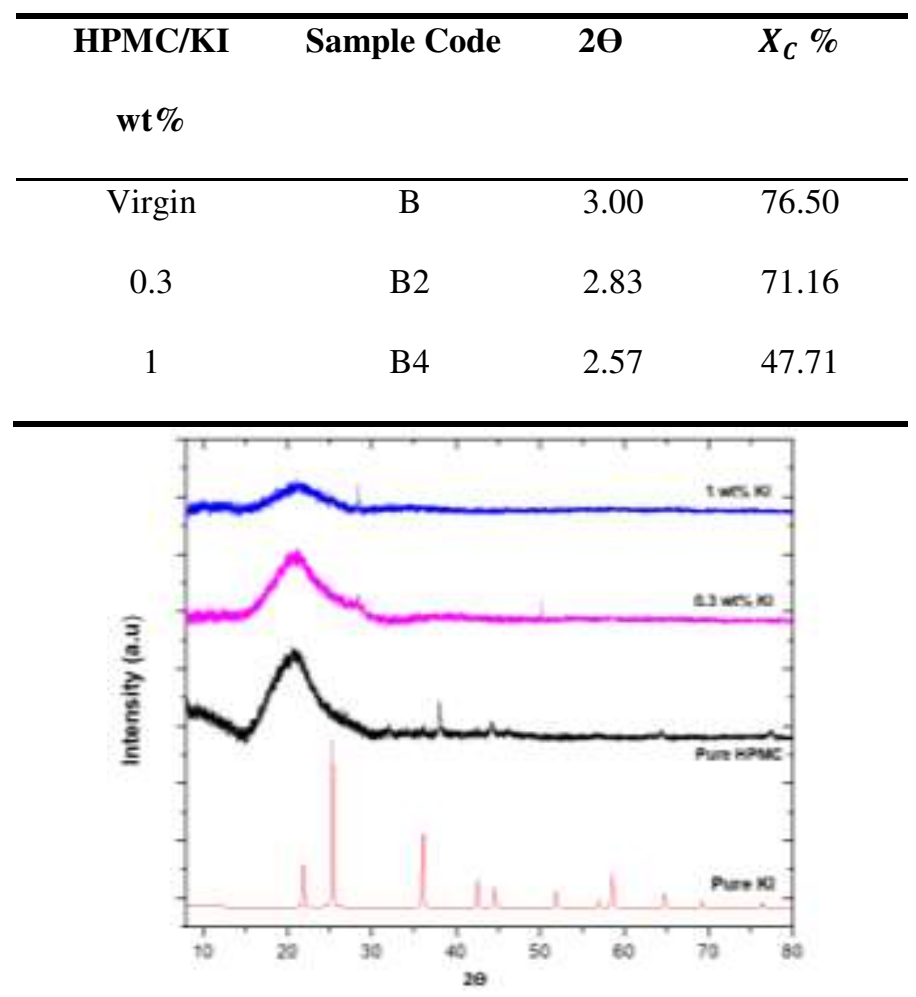

Fig. 12 XRD spectrum of (B) $\mathrm{NaBr}$ salt, (B2) HPMC with $0.3 \mathrm{wt} \% \mathrm{NaBr}$, and (B4) HPMC with 1 wt $\% \mathrm{NaBr}$ of samples

\subsection{FTIR Study}

Figure 13 shows the FT-IR spectra of pure HPMC and HPMC with different wt\% (B, B2, and B4) of composite films appearing in the wavenumber range of $4000-500 \mathrm{~cm}^{-1}$. The fact that the permissible modes of vibration of various functional classes coincide with the IR characteristic bands of pure HPMC film [8-9]. The FT-IR spectra for HPMC showed an absorption band due to a hydroxyl group (-OH) and intermolecular hydrogen bonding in the region from 3449-3847 $\mathrm{cm}^{-1}$. The intensity and interaction of the hydrogen bond in the polymer matrix are measured through hydroxyl absorption [62]. The $\mathrm{OH}$ stretching shifted and broadened to a lower wavenumber as the KI salt concentration increased in complexed films, implying a higher degree of hydrogen 
bonding due to KI salt interaction in the host polymer. An auxiliary broad peak for HPMC was observed at 2908 $\mathrm{cm}^{-1}$, which may be endorsed to symmetric stretching of methyl $\left(-\mathrm{CH}_{3}\right)$ and hydroxypropyl groups $\left(-\mathrm{CH}_{2} \mathrm{CH}\right.$ $\left.\left(\mathrm{CH}_{3}\right) \mathrm{OH}\right)$. A sequential change in the peak was observed for complex films, which is the product of the formation of complexes in the polymer film, which causes band length variation. The C-O bond stretching within the sixmember cyclic ring, asymmetric and symmetric bending vibrations of a methoxy group $\left(\mathrm{O}-\mathrm{CH}_{3}\right)$ will trigger the peaks at $1663 \mathrm{~cm}^{-1}, 1455 \mathrm{~cm}^{-1}$, and $1349 \mathrm{~cm}^{-1}$, respectively. The C-O-C stretching vibrations can be attributed to the characteristic peak at $1046 \mathrm{~cm}^{-1}$ (Fig. B). The asymmetric pyranose ring may be responsible for the peak at $948 \mathrm{~cm}^{-1}$, and the change in the intensity of the peak was observed for the complex films (B2 and B4) as shown in Fig. 13 [7-9].

The effect of dopant salt on the modes of vibrations was seen in terms of a decrease in amplitude, broadening of the bands, and shifting of the bands to lower wavenumbers, as well as the emergence of new peaks in the IR spectra. All of which directly implies the complexation between potassium conducting salt and the host polymer [7-9]. The $\mathrm{OH}$ groups play an important role in directing the crystalline packing [63]. Because of the increase of dopant in the host polymer, crystallization is disrupted due to a significant change in the strength of the $\mathrm{OH}$ groups. Resulting in an increase of a fraction of amorphous material and a decrease in the percentage of crystallinity, simultaneously an increase of Urbach energy. The FTIR results correlate with the observed XRD and Urbach energy results.

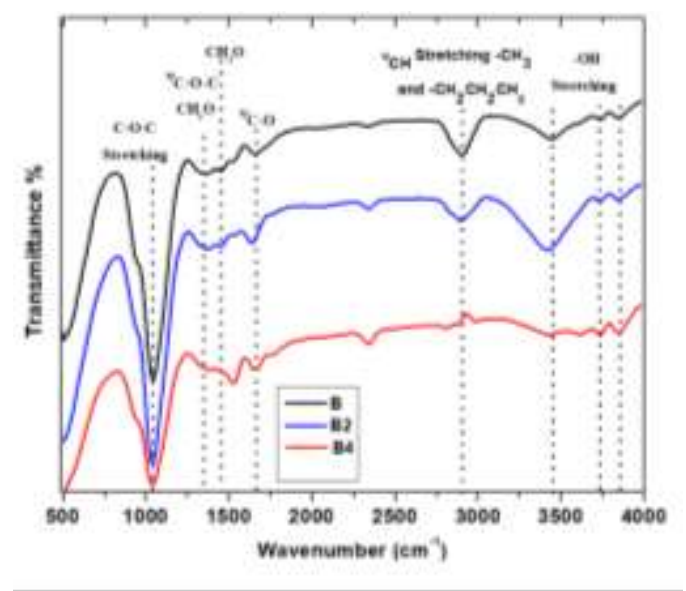

Fig. 13 FT-IR spectrum of pure HPMC and doped HPMC samples

\section{Conclusion}

Herein, Polymer salt-composites with a narrow optical band gap were made by solution casting using Hydroxypropyl Methylcellulose (HPMC). The salt-composite samples transmittance spectra were collected, and 
they showed transparency of more than $85 \%$. The growth of the charge transfer complex in the HPMC host polymer has been attributed to a large shift in the absorption edge to relatively low photon energy. The refractive index plot of the doped films showed a strong dispersion. For the doped HPMC sample with 1wt\% of KI salt, the direct and indirect optical bandgap were shown to be reduced from $5.6 \mathrm{eV}$ to $2.56 \mathrm{eV}$ and 5.86 to $2.5 \mathrm{eV}$ respectively. This reduction is due to the development of the charge transfer complex between the polymer chains which creates several trap sites between the valence and conduction bands. The optical bandgap was exactly estimated using the optical dielectric loss plot while the type of electronic transition was determined using Tauc's approach. It has been discovered that the film with the largest dielectric constant is associated with the shortest optical bandgap. This notable outcome demonstrated the Penn model's validity. Interestingly, the Urbach energy and optical conductivity values of the doped samples were found to be higher indicating a large tail-to-tail and band-to-tail transitions. The broad character of the XRD plot and the decrease in the crystalline percentage of the doped samples relative to pure HPMC suggested a developed amorphous fraction in the doped films. The rise in the amorphous fraction is supported by the increase in the Urbach energy from $556 \mathrm{meV}$ for the virgin HPMC film to $874 \mathrm{meV}$ for the doped HPMC films with $1 \mathrm{wt} \%$ of KI salt. Lastly, the decreased transmittance intensity and shifting of bands in the FTIR spectra of the doped samples facilitate the development of a complex between the HPMC host polymer and the dispersed KI salts.

\section{Acknowledgements}

The authors, Sunilkumar (SK) is thankful to the DST Purse lab, Mangalore University for providing the necessary facilities for the characterization of the samples.

\section{Declaration of interests}

The authors declare that they have no known competing financial interests or personal relationships that could have appeared to influence the work reported in this paper.

\section{Reference}

1. O. G. Abdullah, S. A. Hussen, International Conference on Manufacturing Science and Technology (ICMST), University of Sulaimani, Sulaimani, (2010)

2. R. V. Kulkarni, B. Sa, Electroresponsive Polyacrylamide-grafted-xanthan Hydrogels for Drug Delivery, J Bioact Compat Pol, 24:368, (2009). https://doi.org/10.1177\%2F0883911509104475 
3. P. Chandra Sekhar, P. Naveen Kumar, U. Sasikala, V. V. R. N. Rao, A. K. Sharma, "Investigations on lithium-ion complexed polyvinyl chloride (PVC) solid polymer electrolyte films," IRACST- ESTIJ, 2(5), 908-912, (2012)

4. L. N. Ismail, H. Zulkefle, S. H. Herman, M. Rusop Mahmood, "Influence of doping concentration on dielectric, optical, and morphological properties of PMMA thin films," Advances in Materials Science and Engineering, vol. 2012, Article ID 605673, 5 pages (2012). https://doi.org/10.1155/2012/605673

5. M. R. Ranganath and Blaise Lobo "Experimental investigation of the optical band gap in films of iodine doped polyvinylalcohol - polyvinylpyrrolidone blend" Proceedings of the 52nd DAE Solid State Physics Symposium (India). 52, 495-496, (2007)

6. M. R. Ranganath and Blaise Lobo "Study of UV Visible Absorption Spectra of Aqueous Ferric Chloride Doped Polyvinyl alcohol - Polyvinylpyrrolidone blend films” Proceedings of the International Conference on Condensed Matter Physics (ICCMP-2007), November 25-28, 118 (2007)

7. Y. Prakash, H. Somashekarappa, A. Manjunath, Mahadevaiah, R. Somashekar. Physico-mechanical, AC-conductivity and microstructural properties of $\mathrm{FeCl}_{3}$ doped $\mathrm{HPMC}$ polymer films. Advances in Materials Research, Vol. 2, No. 1, 37-49, (2013). DOI: http://dx.doi.org/10.12989/amr.2013.2.1.037

8. N. SandhyaRania, J. Sannappa, T. Demappa, Mahadevaih. Effects of $\mathrm{CdCl}_{2}$ concentration and gamma irradiation on the structural, thermal and electrical conductivity properties of HPMC polymer electrolyte films. OSR Journal of Applied Physics (IOSR-JAP), Volume 6, Issue 3 Ver. I (May-Jun. 2014), KIPP 30-41.

9. N. SandhyaRania, J. Sannappa, T. Demappa, Mahadevaih. Structural, thermal, and electrical studies of sodium iodide (NaI)-doped hydroxypropyl methylcellulose (HPMC) polymer electrolyte films, Ionics. volume 20, 201-207, (2014). https://doi.org/10.1007/s11581-013-0952-8

10. N. SandhyaRani, M. S. Manjunathab, J. Sannappa, T. Demappa. Studies of Hydrogen Bond between HPMC Doped $\mathrm{CdCl}_{2}$ Polymer using FTIR Technique. Materials Today: Proceedings 5, 22543-22546, (2018). https://doi.org/10.1016/j.matpr.2018.06.626

11. D.L. Kaplan, J. M, D. Mayer, J. Ball, A.L. McCassie, P. Allen, Stenhouse fundamentals of biodegradable polymers, in: C. Ching, D.L. Kaplan, E.L. Thomas (Eds.), Biodegradable Polymers and Packaging, Technomic Pub Co, Lancaster, 1-42. (1993) 
12. K. Van de Velde, P. Kiekens, Biopolymers: overview of several properties and consequences on their applications, Polym. Test. 21 (4), 433-442, (2002). http://dx.doi.org/10.1016/S0142-9418(01)00107-6

13. Shunichiro Ito, Amane Hirose, Madoka Yamaguchi, Kazuo Tanaka, Yoshiki Chujo. Synthesis of Aggregation-Induced Emission-Active Conjugated Polymers Composed of Group 13 Diiminate Complexes with Tunable Energy Levels via Alteration of Central Element. Polymers, 9, 68, (2017). https://dx.doi.org/10.3390\%2Fpolym9020068

14. A. T. Mallajosyula, N. Srivastava, S.S. Kumar Iyer, Baquer Mazhari. Characterization of matrix and isolated organic solar cells. Sol. Energy Mater. Sol. Cells, 94, 1319-1323, (2010). https://doi.org/10.1016/j.solmat.2008.09.027

15. Hofenk-de Graaff J (1981) Central research laboratory for objectsof art and science. Gabriel Metsustroat and 1071 EA, Amsterdam, the Netherlands

16. S. Honary, P. Ebrahimi, N. Emrani, The effect of plasticizer molecular weights and concentrations on water vapor permeability of hydroxy propyl methyl cellulose films, Int J Pharma Bio Sci, 1(2), (2010).

17. I. J. Hardy, W. G. Cook, C. D. Melia, Compression and compaction properties of plasticized high molecular weight hydroxypropyl methylcellulose (HPMC) as a hydrophilic matrix carrier. Inter J Pharm 311(1-2):26-32, (2006). https://doi.org/10.1016/j.ijpharm.2005.12.025

18. Hunter CC, Ingram MD, Na+-ion conducting glasses, Solid State Ionics, 14(1), 31-40, (1984). https://doi.org/10.1016/0167-2738(84)90007-9

19. Bruce PG, Vincent CA, Polymer electrolytes, J Chem Soc Faraday Trans 89:3187-3203, (1993).

20. K. M. Abd El-Kader and A. S. Orabi, "Spectroscopic behavior of poly(vinyl alcohol) films with different molecular weights,” Polymer Testing, vol. 21, no. 5, pp. 591-595, (2002). https://doi.org/10.1016/S0142-9418(01)00129-5

21. K. H. Tagreed, "Refractive index dispersion and analysis of the optical parameters of (PMMA/PVA) thin film,” Journal of AlNahrain University, vol. 16, no. 3, pp. 164-170, 2013.

22. Wu, Y.; Wadia, C.; Ma, W.; Sadtler, B.; Alivisatos, A.P. Synthesis and Photovoltaic Application of Copper (I) Sulfide Nanocrystals. Nano Lett. 2008, 8, 2551-2555. [CrossRef] [PubMed] 
23. Miah, M.J.; Shahabuddin, M.; Karikomi, M.; Salim, M.; Nasuno, E.; Kato, N.; Iimura, K. Structural and Photoelectrical Characterization of Thin Films of a Novel Amphiphilic Oxa[9]helicene Derivative. Bull. Chem. Soc. Jpn. 2016, 89, 203-211.

24. Weiwei, L.; Koen, H.H.; Martijn, M.W.; René, A.J.J. Diketopyrrolopyrrole Polymers for Organic Solar Cells. Acc. Chem. Res. 2016, 49, 78-85.

25. Dong, M.; Dan, S.; Chengmei, Z.; Tao, L.; Bingbing, F.; Lijun, H.; Yan, L.; Wei, J.; Hyosung, C.; Taehyo, K.; et al. High-Performance Solution-Processed Non-Fullerene Organic Solar Cells based on Selenophene-Containing Perylene Bisimide Acceptor. J. Am. Chem. Soc. 2016, 138, 375-380.

26. Elanz Sadeghi, Effect of strong electrolyte containing gelling aids on the solgel transition temperature of Hypromellose 2910, 2019

27. N. KrishnaJyothi, K.K. VenkataRatnam, P. NarayanaMurthy, K. VijayaKumar, Electrical Studi es of Gel Polymer Electrolyte based on PAN for Electrochemical Cell Applications, Volume 3, Issue 1, 2016, Pages 21-30, https://doi.org/10.1016/j.matpr.2016.01.112

28. N Krishna Jyothi, K K Venkataratnam, P Narayana Murty And K Vijaya Kumar Preparation and characterization of PAN-KI complexed gel polymer electrolytes for solid-state battery applications

29. G. Rajesha Shetty, B. L. Rao, S. Asha, Y. Wang, Y. Sangappa, Fiber Polym. 16 (2015) 1734-1741.

30. Aziz, S.B.; Abdulwahid, R.T.; Rsaul, H.A.; Ahmed, H.M. In situ synthesis of CuS nanoparticle with a distinguishable SPR peak in NIR region. J. Mater. Sci. 2016, 27, 4163-4171.

31. Rao, Y.Q.; Chen, S. Molecular Composites Comprising TiO2 and Their Optical Properties. Macromolecules 2008, 41, 4838-4844.

32. B L Lakshmeesha Rao, C S Shivananda, G Rajeev Shetty, K V Harish, R Madhukumar and Y Sangappa. (2018) Influence of UV irradiation on Hydroxy Propyl Methyl Cellulose polymer films. AIP coference proceedings (ICC 2017), 1953, 080011-1-080011-4.

33. A. Wasan, T. Mohammed, and K. Tagreed, "The MR affect on optical properties for poly (Vinyl alcohol) films," Journal of Baghdad for Science, vol. 8, no. 2, pp. 543-550, 2011. 
34. B. Lobo, M. R. Ranganath, T. S. G. Ravi Chandran, G. V. Rao, V. Ravindrachary, and S. Gopal, “Iodine-doped polyvinylalcohol using positron annihilation spectroscopy,” Physical Review B, vol. 59, no. 21, pp. 13693-13698, 1999.

35. H. Zahr El-Deenb and A. I. Hafez, "Physico-chemical stability of pva films doped with Mn2+ ions against weathering conditions," The Arabian Journal for Science and Engineering, vol. 34, no. 1, pp. 13-26, 2009.

36. M. Hamed, H. Sabah, and A. Sarkawt, "Electrical and optical properties of PVA/LiI polymer electrolyte films," Asian Transections on Science and Technology, vol. 1, no. 6, pp. 16-20, 2012

37. A. Juma, E. Arbab, S. Afirca, Synthesis and characterization of CuO-NiO-ZnO mixed metal oxide nanocomposite, J. Alloy. Comp. (2017), https://doi.org/10.1016/j.jallcom.2017.06.288.

38. Abdulwahid, R.T.; Abdullah, O.G.; Aziz, S.B.; Hussein, S.A.; Muhammad, F.F.; Yahya, M.Y. The study of structural and optical properties of PVA: $\mathrm{PbO} 2$ based solid polymer nanocomposites. J. Mater. Sci. 2016, 27, 12112-12118.

39. Guirguis O Wand MoselhyMTH2011 J. Mater. Sci. 46 5775-89

40. Nakabayashi, K.; Takeuchi, Y.; Mori, H. Perylene Bisimide-Based DonorAcceptor Materials Incorporating

41. Aziz, S.B.; Ahmed, H.M.; Hussein, A.M.; Fathulla, A.B.; Wsw, R.M.; Hussein, R.T. Tuningthe absorption of ultraviolet spectra and optical parameters of aluminum doped PVA based solid polymer composites. J. Mater. Sci. 2015, 26, 8022-8028.

42. Abd El-Raheem MM (2007) J Phys Condens Matter 19:216209

43. Aziz, S.B. Modifying Poly(Vinyl Alcohol) (PVA) from Insulator to SmallBandgap Polymer: A Novel Approach for Organic Solar Cells and Optoelectronic Devices. J. Electron. Mater. 2016, 45, 736-745.

44. Abdel-Baset, T.; Elzayat, M.; Mahrous, S. Characterization and Optical and Dielectric Properties of Polyvinyl Chloride/Silica Nanocomposites Films. Int. J. Polym. Sci. 2016, 2016, 1-13.

45. Prasher, S.; Kumar, M.; Singh, S. Electrical and Optical Properties of O6+ Ion Beam-Irradiated Polymers. Int. J. Polym. Anal. Charact. 2014, 19, 204-211. 
46. Saq'an, S.A.; Ayesh, A.S.; Zihlif, A.M.; Martuscelli, E.; Ragosta, G. Physical properties of polystyrene/alum composites. Polym. Test. 2004, 23, 739-745.

47. Mohan, V.M.; Raja, V.; Bhargav, P.B.; Sharma, A.K.; Rao, V.V.R.N. Structural, electrical and optical properties of pure and NaLaF4 doped PEO polymer electrolyte films. J. Polym. Res. 2007, 14, 283-290

48. Ibrahim, S.; Ahmad, R.; Johan, M.R. Conductivity and optical studies of plasticized solid polymer electrolytes doped with carbon nanotube. J. Lumin. 2012, 132, 147-152.

49. N.A. Bakr, A.M. Funde, V.S. Waman, M.M. Kamble, R.R. Hawaldar, D.P. Amalnerkar, S.W. Gosavi, S.R. Jadkar, Determination of the optical parameters of a-Si:H thin films deposited by hot wirechemical vapour deposition technique using transmission spectrum only, Pramana - J. Phys. 76 (2011) 519-531, https://doi.org/10.1007/s12043-011-0024-4.

50. KumarGVand Chandramani R 2009 App. Surfac. Sci. 255 7047-50

51. Siddaiah T, Ojha P, GopalNO, RanuCand NagabhushanaH2018 J. Sci.: Advanc. Mater. Devic. 3 45663

52. Sheela T, Bhajantri R F, Ravindrachary V, Rathod SG, Pujari PK, Poojary B and Somashekar R 2014 Rad. Phys. Chem. 103 45-52

53. Abd El-Kader FH, Gafer SA, Basha A F, Bannan S I and BashaMA F 2010 J. Appl. Polym. Sci. 118 413-20.

54. Z.N. Nanocomposites, M.A. Ditta, M.A. Farrukh, S. Ali, N. Younas, X-ray peak pro filing, optical parameters and catalytic properties of pure and CdS doped, Russ. J.Appl. Chem. 90 (2017), https://doi.org/10.1134/S1070427217010220.

55. Pankaj, S. and Katyal, S.C., J. Phys. D: Appl. Phys., 2007, vol. 40, p. 2115.

56. Nadeem, M. and Ahmed, W., Turk. J. Phys., 2000, vol. 24, p. 651.

57. Biskri, Z.E.; Rached, H.; Bouchear, M.; Rached, D.; Aida, M.S. A Comparative Study of Structural Stability and Mechanical and Optical Properties of Fluorapatite (Ca5(PO4)3F) and Lithium Disilicate (Li2Si2O5) Components Forming Dental Glass-Ceramics: First Principles Study. J. Electron. Mater. 2016, 45, 5082-5095. 
58. K. A. Khadum, "Study the optical constant of cadmium oxide films doped with silver oxide (CdO: Ag2O) in infrared region,” Diala, Jour, vol. 32, pp. 1-15, 2009.

59. Ravindra, N.M.; Ganapathy, P.; Choi, J. Energy gap-refractive index relations in semiconductors-An overview. Infrared Phys. Technol. 2007, 50, 21-29.

60. Yu, L.; Li, D.; Zhao, S.; Li, G.; Yang, K. First principles study on electronic structure and optical properties of ternary GaAs:Bi alloy. Materials 2012, 5, 2486-2497.

61. F. Yakuphanoglu, A. Cukurovali, and I. Yilmaz, "Refractive index and optical absorption properties of the complexes of a cyclobutane containing thiazolyl hydrazone ligand," Optical Materials, vol. 27, no. 8, pp. 1363-1368, 2005.

62. Turhan, K.N.; Ahbaz,F.S.;Guner,A.A Spectrophotometric Study of Hydrogen Bonding in Methylcellulose-based Edible Films Plasticized by Polyethylene Glycol. J. Food Sci. 2001, 66, 59-62.

63. Sjostrom, E. 1981. Wood Chemistry: Fundamentals and applications, Academic Press, New York, 169189. 